\title{
Beyond Strong Coupling in a Multimode Cavity
}

\author{
Neereja M. Sundaresan, Yanbing Liu, Darius Sadri, László J. Szőcs, Devin L. Underwood, \\ Moein Malekakhlagh, Hakan E. Türeci, and Andrew A. Houck" \\ Department of Electrical Engineering, Princeton University, Princeton, New Jersey 08544, USA
}

(Received 20 February 2015; published 29 June 2015)

\begin{abstract}
Here, we report an experimental realization of multimode strong coupling in cavity quantum electrodynamics. This novel regime is achieved when a single artificial atom is simultaneously strongly coupled to a large, but discrete, number of nondegenerate photonic modes of a cavity with coupling strengths comparable to the free spectral range. Our experiment reveals complex quantum multimode dynamics and spontaneous generation of quantum coherence, as evidenced by resonance fluorescence spanning many modes and ultranarrow linewidth emission. This work opens a new avenue for future experiments in light-matter interactions and poses a challenge to current theoretical approaches to its study.

DOI: 10.1103/PhysRevX.5.021035

Subject Areas: Condensed Matter Physics,

Photonics, Quantum Physics
\end{abstract}

The study of light-matter interaction has seen a resurgence in recent years, stimulated by highly controllable, precise, and modular experiments in cavity quantum electrodynamics (QED) [1]. The achievement of strong coupling [2-4], where the coupling between a single atom and fundamental cavity mode exceeds the decay rates, was a major milestone that opened the doors to a multitude of new investigations $[5,6]$.

Here, we investigate multimode strong coupling (MMSC) $[7,8]$, where the coupling is comparable to the free spectral range (FSR) of the cavity; i.e., the rate at which a qubit can absorb a photon from the cavity is comparable to the roundtrip transit rate of a photon in the cavity. We realize, via the circuit QED architecture, an experiment accessing the MMSC regime and report remarkably widespread and highly structured resonance fluorescence. The observed drive dependence of the width, height, and position of the fluorescence peaks cannot be explained by cavity enhancement of sidebands observed in the single-mode regime [9]. As expounded below, our observations reveal a generation of coherence across multiple frequencies mediated by a single qubit and necessitate a multimode analysis. Beyond the novel phenomena presented here, the access to the MMSC regime opens up a new direction of exploration that is of interest both theoretically and experimentally.

Interest in going beyond strong coupling has focused on the ultrastrong-coupling limit, where the breakdown of the rotating-wave approximation for the light-matter interaction results in excitation nonconserving terms [10-13]. In

\footnotetext{
* Corresponding author. aahouck@princeton.edu
}

Published by the American Physical Society under the terms of the Creative Commons Attribution 3.0 License. Further distribution of this work must maintain attribution to the author(s) and the published article's title, journal citation, and DOI. contrast, the direction which we pursue is the simultaneous strong coupling of the qubit to numerous modes, leading to qubit-mediated mode-mode interactions and nonlinear quantum dynamics not present in the single-mode problem. Thus, MMSC demonstrates a qualitatively new domain, intermediate between the quantum mechanics of systems with a small number of degrees of freedom and full continuum quantum field theory in free space. Unlike the traditional spin-boson problem that involves a bosonic continuum with an algebraic bath spectral function of the type $J(\omega)=\alpha \omega^{s}$, the MMSC regime is described by a structured spectral function with an infinite number of finite-width peaks. While the focus in the spin-boson problem is on the ensuing strong spin-bath correlations [7,14], in the MMSC regime the focus is on the resulting complex temporal dynamics of the bosonic degrees of freedom. It was pointed out [8] that the quench dynamics in this regime feature pulsed emission characterized by the cavity round-trip time, instead of Purcell modified pure exponential decay or damped Rabi oscillations characterizing the single-cavity-mode problem. Driving such a system is hence expected to lead to an entirely new quantum dynamical regime that may feature frequency correlations that are unique to the multimode nature of the coupling. Finally, in contrast to the single-mode problem [15], the multimode problem is not known to be integrable. Therefore, this regime requires new theoretical techniques for its further exploration.

The closed system is described by the Hamiltonian

$$
H=\frac{\hbar}{2} \omega_{a} \sigma_{z}+\sum_{m}\left[\hbar \omega_{m} a_{m}^{\dagger} a_{m}+\hbar g_{m}\left(\sigma^{+}+\sigma^{-}\right)\left(a_{m}^{\dagger}+a_{m}\right)\right] \text {, }
$$

where $\omega_{a}$ is the qubit frequency, $\sigma_{x, y, z}$ are Pauli pseudospin operators, and $m$ represents cavity-mode number. Here, $a_{m}^{\dagger}$ $\left(\sigma^{+}\right)$and $a_{m}\left(\sigma^{-}\right)$are mode (qubit) raising and lowering 
operators. The coupling strength of the qubit to the $m$ th harmonic is $g_{m}=g_{0} \sqrt{m+1}$, where $g_{0}$ is the coupling constant to the fundamental cavity mode [16].

To achieve MMSC, the coupling between qubit and cavity must be comparable to the free spectral range, which is made possible through use of a long cavity. Previously, long cavities have been used for novel comb generation, with a Kerr nonlinearity provided by the bulk medium [17]. Other unique multimode platforms have also been studied, including systems where a quasidegenerate set of transverse modes of a cavity are coupled to ensembles of atoms in cavity QED [18-20], and degenerate lumped LC resonators are coupled to qubits in circuit QED [21]. In contrast, here the nonlinearity is provided by a single qubit that is coupled strongly to a large set of longitudinal modes of a very long coplanar waveguide cavity.

In our setup, the coupling to the fundamental mode [22] $g_{0}$ scales with cavity length $L$ as $g_{0} \sim 1 / L$. Holding constant the qubit frequency, we are interested in the $n$th harmonic of the cavity which is nearly resonant with the qubit. The ratio of the coupling for this mode to the FSR then scales as $g_{n} /$ FSR $\sim \sqrt{L}$ (see the Supplemental Material [23]). As there are many modes simultaneously interacting with the qubit, many modes can be coupled to each other during the typical lifetime of an excitation in the system, with the effective qubit-mediated interaction rate between a pair of modes being $g_{m} g_{n} /(n-m)$ FSR, leading to a delocalization of the excitation across many modes.

We design a very low-frequency cavity such that the $\omega_{a}$ of a standard qubit falls near a high harmonic (approximately 50th-75th) of the cavity, unlike traditional circuit QED experiments. Additionally, this approach allows us to adapt to the typical measurement range $(3-8 \mathrm{GHz})$ of existing circuit QED setups. The superconducting microwave cavity comprises a $0.68-\mathrm{m}$ transmission line with identical capacitors on either end, resulting in a fundamental frequency $\omega_{0} / 2 \pi \sim 92 \mathrm{MHz}$ (see Fig. 1 and the Supplemental Material for details [23]). The harmonics of the cavity are uniformly separated, a constant FSR, which empirically is the result of the essentially fixed dielectric constant of sapphire in the microwave frequency range.

We couple a flux-tunable transmon qubit [24] near one end of the resonator, an antinode for all the modes of the cavity, and achieve a qubit-mode coupling strength for the 75th cavity harmonic exceeding $30 \mathrm{MHz}$. Coherent drive, at frequency $\omega_{d}$, is introduced only via the input port [Fig. 1(b)], while radiative decay of cavity photons occurs via both input and output ports. A unitary transformation maps the coherent drive from the cavity to the qubit, yielding a qubit driving term $\Omega \cos \left(\omega_{d} t\right)\left(\sigma^{+}+\sigma^{-}\right)$, with $\Omega$ the Rabi drive amplitude (see the Supplemental Material [23]). The mode linewidths $\kappa_{m} / 2 \pi$ span from $0.5-3 \mathrm{MHz}$ to provide reasonable separation between modes. The qubit spontaneous decay rate $\gamma / 2 \pi$ is of the order of $1.6 \mathrm{MHz}$, due to the multimode Purcell effect [16].
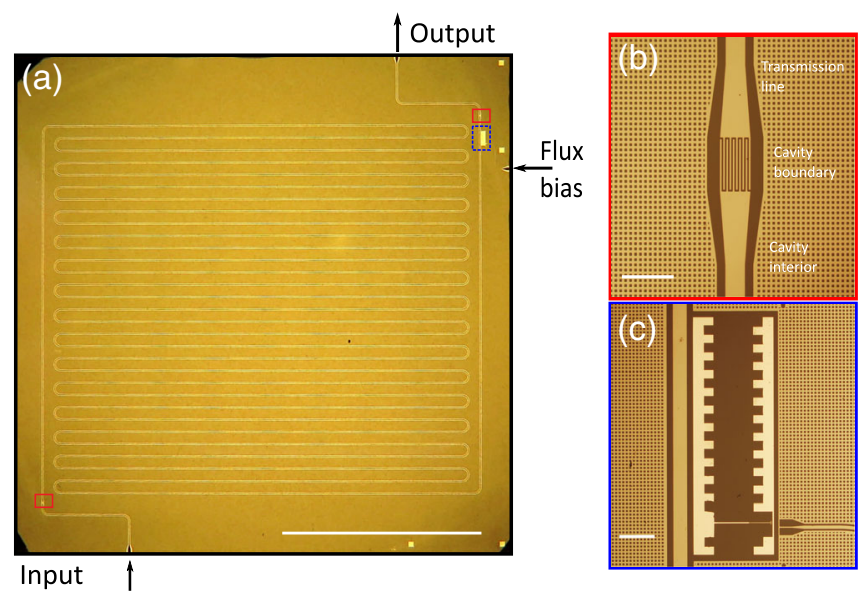

FIG. 1. Device for achieving MMSC. (a) A low fundamental frequency $\omega_{0} / 2 \pi \sim 92 \mathrm{MHz}$ is realized by fabricating a meandering $0.68-\mathrm{m}$ coplanar waveguide resonator on a $25 \times 25-\mathrm{mm}$ sapphire substrate. The capacitive coupling at the input and output ports allows coupling of radiation into and out of the resonator, at rates $\kappa_{m} / 2 \pi \sim 0.5-3 \mathrm{MHz}$. Red rectangles show coupling capacitors. The blue rectangle encloses the qubit. (b) Symmetric interdigitated capacitors define the cavity boundaries and couple radiation to the transmission lines. (c) A transmon qubit is capacitively coupled to the center pin near the output capacitor, an antinode for all modes. Superconducting quantum interference device geometry allows for tuning qubit frequency $\omega_{a}$ via the flux bias line (shown) or the external magnet [24]. The gridlike pattern in the ground plane pins flux vortices. Scale bars denote (a) $10 \mathrm{~mm}$, (b) $100 \mu \mathrm{m}$, and (c) $100 \mu \mathrm{m}$.

Measuring transmission while tuning the qubit frequency reveals that the qubit strongly couples to a vast number of modes [Fig. 2(a)] [3]. In fact, we are able to follow the trail of avoided crossings for several $\mathrm{GHz}$ as the qubit energy tunes through the uniformly dense mode structure. The smooth transitions between subsequent avoided crossings show that the qubit is perpetually near resonance with some mode, and as such the dispersive approximation is never valid. In Figs. 2(b) and 2(c), the marked difference in the magnitude of the avoided crossings, and thus coupling strength, is apparent. We fit the avoided crossings in Fig. 2 to the first excitation manifold of the multimode Jaynes-Cummings Hamiltonian to find $g_{0} / 2 \pi \sim 3.75 \mathrm{MHz}$, giving rise to $g_{75} / 2 \pi \sim 32 \mathrm{MHz}$ (see the Supplemental Material [23]).

Within the rich domain of MMSC, we first focus our attention on resonance fluorescence, or the response of a qubit to coherent drive. Strong resonant driving of a qubit in free space results in emission forming a three-peaked structure known as the Mollow triplet, composed of a center peak and two symmetric sidebands [25]. The sidebands are displaced linearly from the central peak by the Rabi amplitude $\Omega$, and their widths are proportional to the system dissipation [26]. This phenomenon, also observed experimentally with superconducting qubits [27], is typically explained using the dressed-state picture approach [28]. Coupling of a qubit to a single-mode cavity strongly 

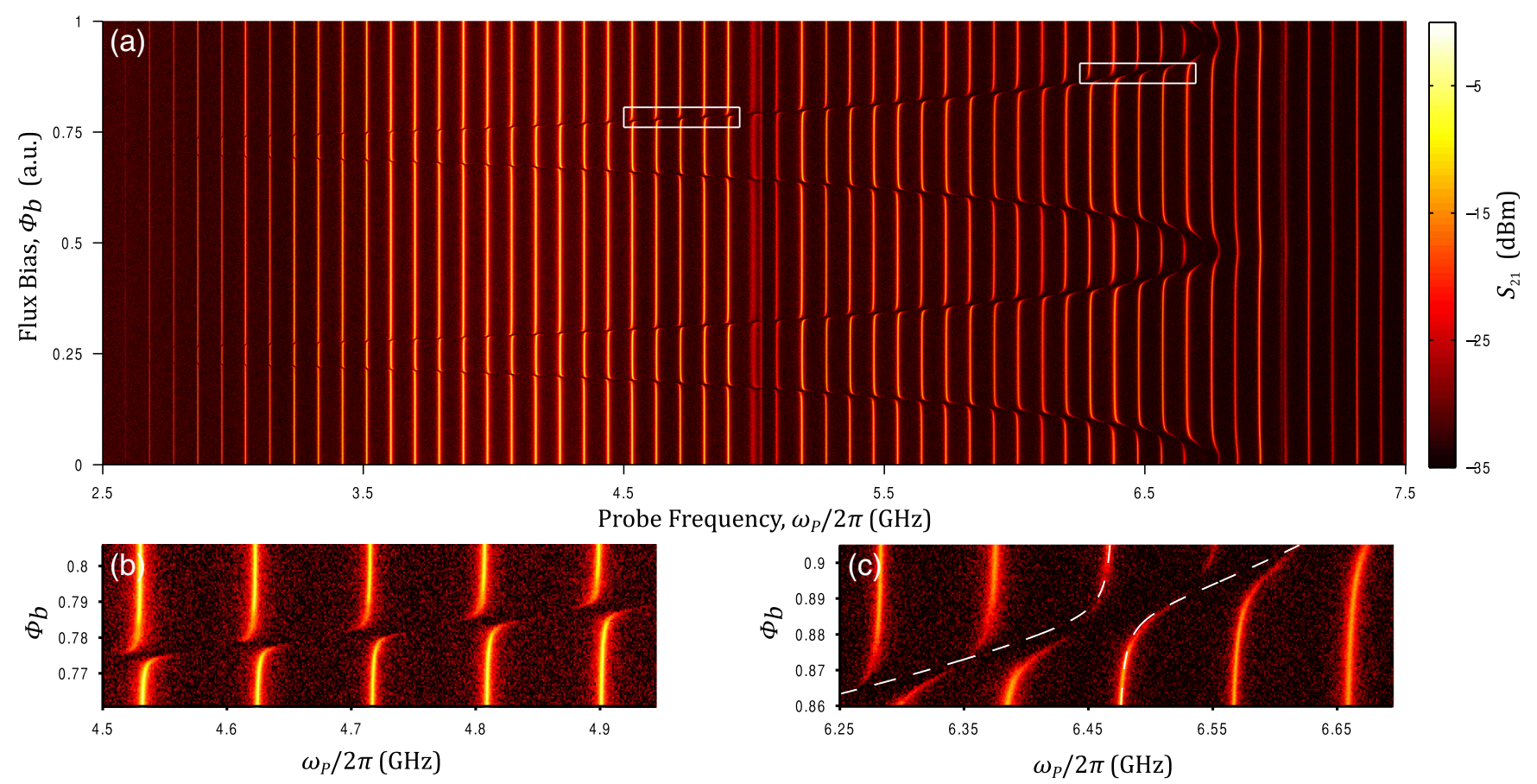

FIG. 2. Demonstrating multimode strong coupling. (a) Low power cavity transmission as qubit frequency is tuned with an applied flux bias. Vacuum Rabi avoided crossings are visible as the qubit is tuned into resonance with each mode (bright vertical lines spaced by approximately $92 \mathrm{MHz}$ ). It is apparent that the vacuum Rabi splitting is comparable with the free spectral range (mode spacing), thus indicating that MMSC has been achieved. White rectangles are shown in (b) and (c). The coupling $g_{m}$ grows with $\mathrm{m}: g_{0} \sqrt{m+1}$. (c) A single-mode fit of the splittings (white dashed line) is not accurate, as evidenced by the strongly shifted states near the central avoided crossing. A fit to the first manifold of the multimode Jaynes-Cummings incorporates all nearby polariton states, yielding $g_{0} \sim 3.75 \mathrm{MHz}$.

modifies the Mollow triplet [29-31]. For weak coupling, the cavity passively filters the fluorescence, while for strong coupling, the sideband width is proportional to the coupling rather than the system dissipation $[9,26]$.

In Fig. 3, we observe fluorescence across more than 50 modes, when the drive and qubit are resonant with a high harmonic of the cavity. Enhanced fluorescence is observed at mode $m$ when it is resonant with the Rabi sideband. As the displacement of Mollow sidebands is proportional to drive amplitude $\Omega$, this occurs [9] when detuning from the $m$ th mode $\Delta_{m} \equiv \omega_{m}-\omega_{d} \approx \Omega$. For far-detuned modes, the drive power needed to reach the peak fluorescence at these modes $P_{d}^{\text {peak }} \approx \Omega^{2}$, and hence $P_{d}^{\text {peak }} \approx \Delta_{m}^{2}$ [Fig. 3(b)].

At fixed drive power, emission is spread over many modes, with the simultaneous enhancement at many nearby modes, differentiating the multimode fluorescence from the single-mode case. With varying drive power, far-detuned modes exhibit a multilobed fluorescence structure [e.g., Fig. 3(c)]. From Fig. 3(d), the drive power which results in the peak of the secondary lobe approaches twice that of the first lobe for increasing detuning $\Delta_{m}$, indicating the second lobe originates from two-photon interactions. These twophoton enhancements, resulting from higher-order interaction vertices $\sigma^{+} a_{m}^{2}$, are of strength $g_{m}^{2} / \Delta_{m}$, which in MMSC exceed the decay rates. Two-photon processes in single-mode cavities, where $\Omega=2 \Delta_{m}$, have previously been observed [32,33]. For MMSC, the multiphoton processes can progress via combinations of many different modes. The resonance condition for single- and multiphoton processes is thus shifted away from $\Omega=p \Delta_{m}$, for $p \in \mathbb{Z}$ (see the Supplemental Material [23]).

Multimode effects become increasingly dominant as we approach modes closer to the qubit, with the appearance of a different intricate multilobed structure [see Figs. 3(e)-3(g)] and the drive powers of brightest fluorescence deviating from the quadratic fit [see the inset of Fig. 3(b)]. These effects arise when qubit-induced interactions between modes $g_{m} g_{n} /(n-m) \mathrm{FSR}$, for $n$ and $m$ near the drive, are comparable to the direct qubit-mode interaction $g_{m}$. Competition between these coherent processes led to the rich phenomena we see in the experiment.

When the mode resonant with the qubit, the center mode, is very weakly driven, there is no incoherent scattering to the other modes. However, at a higher drive power, fluorescence appears simultaneously at multiple nearby modes [three of which are displayed in Figs. 3(e)-3(g)]. In addition to detecting bright fluorescence at these nearby modes (three on either side of the center mode), we also observe incoherent scattering at the center mode itself. Furthermore, there is a change in emission frequency at each of these modes, approaching the bare cavity mode from above with increasing drive power. This shift is 


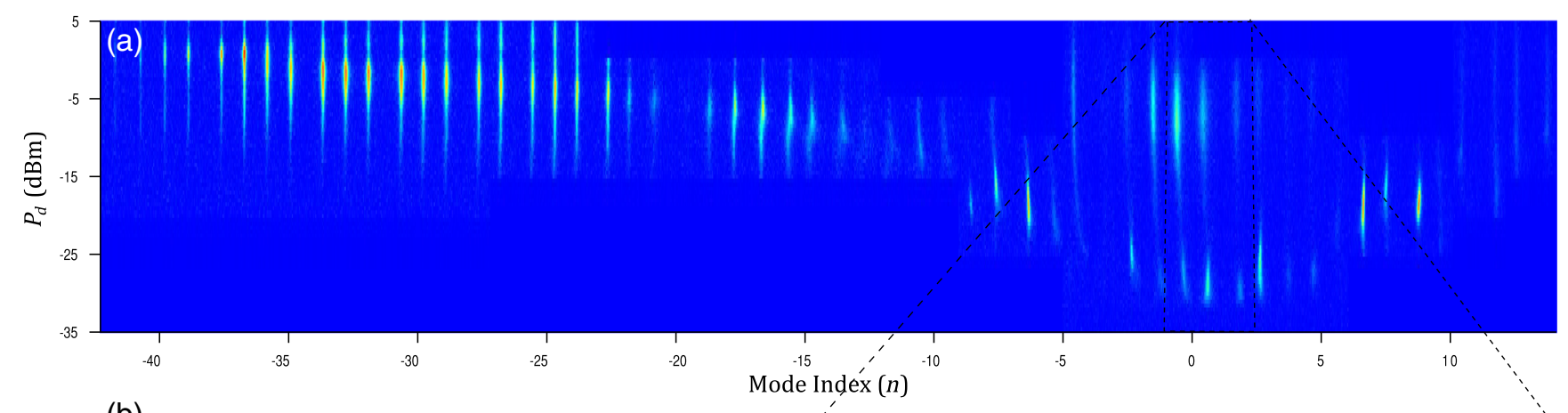

(b)
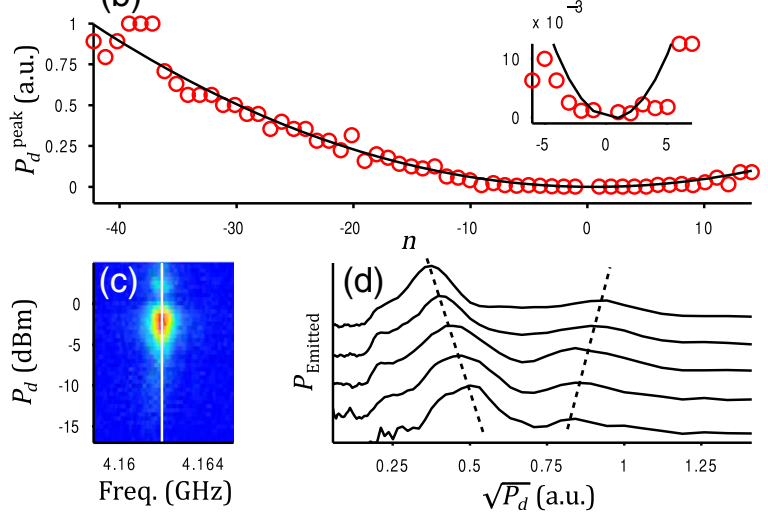
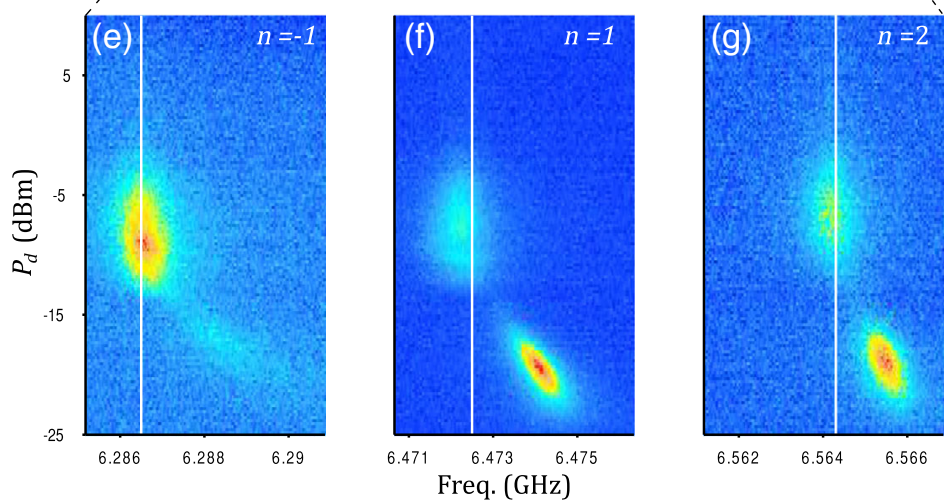

FIG. 3. Multimode resonance fluorescence. (a) Power spectrum for varying drive power $P_{d}$, when $\omega_{d}=\omega_{a}=\omega_{74}$, measured at more than 50 modes. Mode index $n$ is the relative detuning from the drive mode $n=m-74$. Power is measured in a 10-MHz window around each mode, excluding the driven mode $(n=0)$. Increasing drive power unveils fluorescence at farther-detuned modes, strong emission from multiphoton processes, and complex multilobed fluorescence spectra. (b) The drive power corresponding to peak fluorescence for each far-detuned mode shows a quadratic dependence on detuning, suggesting this fluorescence is due to cavity-enhanced sideband fluorescence [9]. Modes near the drive frequency (see the inset) do not follow the quadratic fit due to strong multimode interaction. (c) Detailed plot of $n=-30$ from (a) shows a multilobed structure in fluorescence spectrum. Bare mode frequency is denoted with a vertical white line. For (d) $-(\mathrm{g}), \omega_{d}=\omega_{a}=\omega_{68}$. (d) We explore the difference between the two lobes seen in (c) for several modes ( $n=-36,-34,-32,-30,-28$, from bottom to top with individual curves vertically displaced for clarity) by measuring the total fluorescence power in a 6-MHz window while varying drive power. The drive powers corresponding to peak fluorescence move oppositely with mode number for the two lobes. (e)-(g) For modes near the drive $(n=-1,1,2)$, strong multimode interaction further yields a more complex multilobed structure. Bare cavity-mode frequencies are denoted with vertical white lines.

qualitatively the same for modes on either side of the drive, and cannot be attributed to a simple ac Stark shift.

As drive power is further increased, the simultaneous fluorescence at the nearby modes fades away. At an even higher power, the emission at these modes reappears, but at the bare cavity-mode frequencies. This power coincides with that at which the far-detuned modes fluoresce, suggesting the emergence of qubit-mediated multimode correlation.

A remarkable observation of our experiment is the appearance of ultranarrow linewidths in emission. The fluorescence at far-detuned modes, captured in Fig. 3, has linewidths that are roughly equal to those of the bare modes. For modes nearest the qubit, the fluorescence linewidth is smaller than $\kappa_{m}$, e.g., for the nearest mode above being respectively $650 \mathrm{kHz}$ vs $1.1 \mathrm{MHz}$. Detuning the drive from the center mode unveils here a linewidth narrowing by over an order of magnitude to $65 \mathrm{kHz}$ [Fig. 4(a)]. The narrowest fluorescence occurs when the drive is detuned by $2 \mathrm{MHz}$ from the center mode.
We attribute this narrowing to the spontaneous generation of coherence arising from the coupling of many dressed states through a common vacuum. Such behavior has been predicted and observed in atoms with multiple closely spaced bare atomic levels coupled to vacuum or a single mode of a cavity [34-36], where the theoretical analysis is aided by the simpler dressed-state structure. In our setup, unlike the single-mode case, the number of states in a single excitation manifold is not a constant but rather grows as $\left(\begin{array}{c}N+M-1 \\ M-1\end{array}\right)+\left(\begin{array}{c}N+M-2 \\ M-1\end{array}\right)$, where $M$ is the number of modes and $N$ is the excitation manifold. The rapid growth of the number of states per manifold hinders a simplified analysis via the dressed-state picture, but makes possible the near resonance of many level spacings (Bohr frequencies), leading to a collective enhancement of the coherences from their mutual couplings. An external coherent drive allows us to dynamically access regions of the dressed 


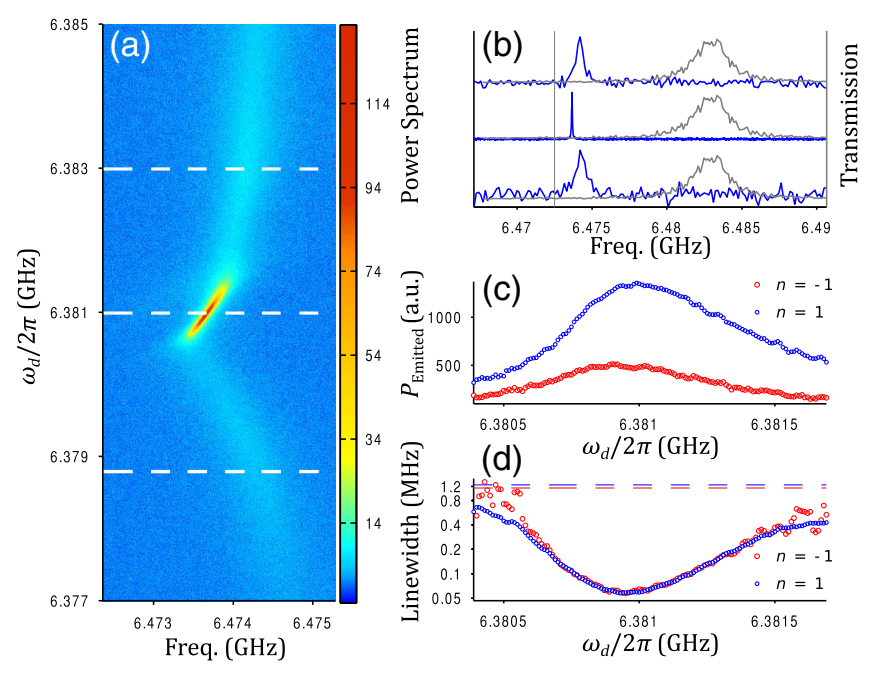

FIG. 4. Ultranarrowing of fluorescence. (a) Power spectrum $(n=1)$ for varying drive frequency $\left(\omega_{d} / 2 \pi\right)$, at fixed drive power, reveals linewidth narrowing. Minimum linewidth $65 \mathrm{kHz}$ corresponds to $\left(\omega_{d}-\omega_{68}\right) / 2 \pi=2 \mathrm{MHz}$. (b) Normalized spectra along dashed lines in (a) are plotted in blue and vertically displaced. For comparison, low power transmission is plotted in solid gray lines and the bare mode frequency is denoted with a vertical gray line. (c) Emitted power (over approximately 3-MHz bandwidth) vs $\omega_{d} / 2 \pi$ shows simultaneous brightening of $n= \pm 1$ modes. (d) Fluorescence linewidths vs $\omega_{d} / 2 \pi$. Linewidths vary concurrently for $n= \pm 1$ and are inversely proportional to emitted power. For comparison, dashed lines show $\kappa / 2 \pi$.

states with large generated coherences, as is evident in the drive dependence of the narrowing in Fig. 4.

In the region where narrowing is observed, the linewidth and steady-state photon number are observed to be inversely proportional, as has been previously suggested for the case of a single mode [37]. For our multimode cavity, the narrowing is in fact observed simultaneously for multiple modes at the same drive frequency, as depicted in Figs. 4(c) and 4(d) for the two modes nearest the drive. While the modes directly neighboring the center mode exhibit the sharpest linewidths of $65 \mathrm{kHz}$, the next-nearest modes also display a narrowed fluorescence of approximately $300 \mathrm{kHz}$. The multilobed power-dependent structure of fluorescence also appears at the optimal detuned drive frequency; however, the linewidth of the second lobe is of order $\kappa_{m}$, for the nearby modes. For more distant modes, while fluorescence follows a similar $\Delta_{m}^{2}$ dependence, there is no narrowing apparent.

The drive-dependent narrowing is not unique to these described experimental parameters. When we translate the drive and qubit to another set of modes, we observe equally narrow linewidth fluorescence, which indicates that the spontaneous generation of coherence is generic in this system. Furthermore, while the qubit is essential for establishing the coherence, the effective Rabi rate is very insensitive to qubit frequency and thus the narrowing is as well (see the Supplemental Material for details [23]); the narrowed fluorescence produced with the detuned drive remains even as the qubit frequency is tuned over $150 \mathrm{MHz}$ away from the center mode.

As an analytical solution of the quantum multimode dynamics is generally inaccessible, we resort to the Monte Carlo wave function approach to simulate a twomode system. To compute two time-correlation functions such as the power spectrum $\int_{-\infty}^{\infty} e^{-i \omega \tau}\left\langle a^{\dagger}(\tau) a(0)\right\rangle d \tau$, we have to employ a two-step procedure [38], equivalent to the quantum regression theorem at the expense of a large number of quantum walks. The produced spectrum for the two-mode system qualitatively agrees with our experimental observation; namely, spectral narrowing appears for a range of Rabi rate $\Omega$, albeit the factor of narrowness is much less than what we observe, and the linewidth is inversely proportional to the photon number.

A unique consequence of the longitudinal multimode configuration is that modes symmetric around the drive will be simultaneously enhanced, leading to much brighter fluorescence than that of a single mode. We gain intuition about the coenhancement by comparing photon number dependences for single- and two-mode systems. Our analysis reveals a dramatic difference, with photon number being proportional and inversely proportional to qubit relaxation rate $\gamma$ in the single-mode and symmetric two-mode cases, respectively. This contrasting dependence arises from additional qubit-mode coupling terms that map both qubit-state flips directly to photon creation in the symmetric two-mode case, which circumvents the necessity of dressed-state population inversion as in the standard single-mode model (see the Supplemental Material for details [23]). With more strongly interacting modes, unitary dynamics in the closed system play an even more important role. Thus, a multimode model is a fundamentally better representation of our experiment than the single-mode model.

In this paper, we realized the multimode strong-coupling regime of circuit QED. We demonstrated the creation of steady states with long-lived quantum coherence, as evidenced by multimode resonance fluorescence with ultranarrow linewidths. Dynamically generated quantum correlations between modes in this driven dissipative system are expected to lead to qualitatively new physics [39]. While this new complex regime poses many theoretical challenges, precise circuit QED experiments can guide this exploration. We hope that access to multimode strong coupling will lead to many new advancements in circuit QED and our understanding of light-matter interactions.

We thank C. Eichler, S. M. Girvin, J. Koch, D. I. Schuster, W. E. Shanks, S. J. Srinivasan, and J. M. Taylor for helpful conversations and technical support. This work was supported by the U.S. Army Research Office (W911NF-11-1-0086), the U.S. National Science Foundation through the Princeton Center for Complex Materials (DMR-0819860) and CAREER awards (Grants No. DMR-0953475 and No. DMR-1151810), the David 
and Lucile Packard Foundation, and the Department of Defense through the NDSEG program. There are no competing financial interests.

Note added.-While this manuscript was under review, one of the co-authors, Darius Sadri, passed away unexpectedly.

[1] S. Haroche and J. Raimond, Exploring the quantum: Atoms, cavities, and photons, Oxford Graduate Texts (Oxford University Press, Oxford, England, 2006).

[2] R. J. Thompson, G. Rempe, and H. J. Kimble, Observation of Normal-Mode Splitting for an Atom in an Optical Cavity, Phys. Rev. Lett. 68, 1132 (1992).

[3] A. Wallraff, D. I. Schuster, A. Blais, L. Frunzio, R.-S. Huang, J. Majer, S. Kumar, S. M. Girvin, and R. J. Schoelkopf, Strong coupling of a single photon to a superconducting qubit using circuit quantum electrodynamics, Nature (London) 431, 162 (2004).

[4] J. P. Reithmaier, G. Sęk, A. Löffler, C. Hofmann, S. Kuhn, S. Reitzenstein, L. V. Keldysh, V. D. Kulakovskii, T. L. Reinecke, and A. Forchel, Strong coupling in a single quantum dot-semiconductor microcavity system, Nature (London) 432, 197 (2004).

[5] H. Mabuchi and A. C. Doherty, Cavity quantum electrodynamics: Coherence in context, Science 298, 1372 (2002).

[6] R. J. Schoelkopf and S. M. Girvin, Wiring up quantum systems, Nature (London) 451, 664 (2008).

[7] D. J. Egger and F. K. Wilhelm, Multimode Circuit Quantum Electrodynamics with Hybrid Metamaterial Transmission Lines, Phys. Rev. Lett. 111, 163601 (2013).

[8] D. O. Krimer, M. Liertzer, S. Rotter, and H. E. Türeci, Route from spontaneous decay to complex multimode dynamics in cavity QED, Phys. Rev. A 89, 033820 (2014).

[9] H. Kim, T. C. Shen, K. Roy-Choudhury, G. S. Solomon, and E. Waks, Resonant Interactions between a Mollow Triplet Sideband and a Strongly Coupled Cavity, Phys. Rev. Lett. 113, 027403 (2014).

[10] T. Niemczyk et al., Circuit quantum electrodynamics in the ultrastrong-coupling regime, Nat. Phys. 6, 772 (2010).

[11] A. A. Anappara, S. De Liberato, A. Tredicucci, C. Ciuti, G. Biasiol, L. Sorba, and F. Beltram, Signatures of the ultrastrong light-matter coupling regime, Phys. Rev. B 79, 201303 (2009).

[12] P. Forn-Díaz, J. Lisenfeld, D. Marcos, J. J. García-Ripoll, E. Solano, C. J. P. M. Harmans, and J. E. Mooij, Observation of the Bloch-Siegert Shift in a Qubit-Oscillator System in the Ultrastrong Coupling Regime, Phys. Rev. Lett. 105, 237001 (2010).

[13] Y. Todorov, A. M. Andrews, R. Colombelli, S. De Liberato, C. Ciuti, P. Klang, G. Strasser, and C. Sirtori, Ultrastrong Light-Matter Coupling Regime with Polariton Dots, Phys. Rev. Lett. 105, 196402 (2010).

[14] A. J. Leggett, S. Chakravarty, A. T. Dorsey, M. P. A. Fisher, A. Garg, and W. Zwerger, Dynamics of the dissipative two-state system, Rev. Mod. Phys. 59, 1 (1987).
[15] D. Braak, Integrability of the Rabi Model, Phys. Rev. Lett. 107, 100401 (2011).

[16] A. A. Houck et al., Controlling the Spontaneous Emission of a Superconducting Transmon Qubit, Phys. Rev. Lett. 101, 080502 (2008).

[17] R. P. Erickson, M. R. Vissers, M. Sandberg, S. R. Jefferts, and D.P. Pappas, Frequency Comb Generation in Superconducting Resonators, Phys. Rev. Lett. 113, 187002 (2014).

[18] S. Gopalakrishnan, B. L. Lev, and P. M. Goldbart, Atom-light crystallization of Bose-Einstein condensates in multimode cavities: Nonequilibrium classical and quantum phase transitions, emergent lattices, supersolidity, and frustration, Phys. Rev. A 82, 043612 (2010).

[19] A. Wickenbrock, M. Hemmerling, G. R. M. Robb, C. Emary, and F. Renzoni, Collective strong coupling in multimode cavity QED, Phys. Rev. A 87, 043817 (2013).

[20] P. Horak, H. Ritsch, T. Fischer, P. Maunz, T. Puppe, P. W. H. Pinkse, and G. Rempe, Optical Kaleidoscope Using a Single Atom, Phys. Rev. Lett. 88, 043601 (2002).

[21] D. C. McKay, R. Naik, P. Reinhold, L. S. Bishop, and D. I. Schuster, High-Contrast Qubit Interactions Using Multimode Cavity QED, Phys. Rev. Lett. 114, 080501 (2015).

[22] C. Cohen-Tannoudji, J. Dupont-Roc, and G. Grynberg, Atom-Photon Interactions: Basic Processes and Applications (Wiley-Interscience, New York, 1992).

[23] See Supplemental Material at http://link.aps.org/ supplemental/10.1103/PhysRevX.5.021035 for additional experimental details and results, detailed analytical discussion and Monte Carlo wave function simulation results.

[24] J. Koch, T. M. Yu, J. Gambetta, A. A. Houck, D. I. Schuster, J. Majer, A. Blais, M. H. Devoret, S. M. Girvin, and R. J. Schoelkopf, Charge-insensitive qubit design derived from the cooper pair box, Phys. Rev. A 76, 042319 (2007).

[25] B. Mollow, Power spectrum of light scattered by two-level systems, Phys. Rev. 188, 1969 (1969).

[26] C. M. Savage, Resonance Fluorescence Spectrum of an Atom Strongly Coupled to a Cavity, Phys. Rev. Lett. 63, 1376 (1989).

[27] O. Astafiev, A. M. Zagoskin, A. A. Abdumalikov, Yu. A. Pashkin, T. Yamamoto, K. Inomata, Y. Nakamura, and J. S. Tsai, Resonance fluorescence of a single artificial atom, Science 327, 840 (2010).

[28] C. Cohen-Tannoudji and S. Reynaud, Dressed-atom description of resonance fluorescence and absorption spectra of a multi-level atom in an intense laser beam, J. Phys. B 10, 345 (1977).

[29] A. Muller, E. B. Flagg, P. Bianucci, X. Y. Wang, D. G. Deppe, W. Ma, J. Zhang, G. J. Salamo, M. Xiao, and C. K. Shih, Resonance Fluorescence from a Coherently Driven Semiconductor Quantum Dot in a Cavity, Phys. Rev. Lett. 99, 187402 (2007).

[30] C. Lang et al., Observation of Resonant Photon Blockade at Microwave Frequencies Using Correlation Function Measurements, Phys. Rev. Lett. 106, 243601 (2011).

[31] G. Oelsner, P. Macha, O. V. Astafiev, E. Il'ichev, M. Grajcar, U. Hübner, B. I. Ivanov, P. Neilinger, and H.-G. Meyer, Dressed-State Amplification by a Single Superconducting Qubit, Phys. Rev. Lett. 110, 053602 (2013). 
[32] W. Lange, G. S. Agarwal, and H. Walther, Observation of Two-Photon Decay of Rydberg Atoms in a Driven Cavity, Phys. Rev. Lett. 76, 3293 (1996).

[33] Y. Ota, S. Iwamoto, N. Kumagai, and Y. Arakawa, Spontaneous Two-Photon Emission from a Single Quantum Dot, Phys. Rev. Lett. 107, 233602 (2011).

[34] P. Zhou and S. Swain, Ultranarrow Spectral Lines via Quantum Interference, Phys. Rev. Lett. 77, 3995 (1996).

[35] M. Kiffner, M. Macovei, J. Evers, and C. H. Keitel, Vacuum-induced processes in multilevel atoms, Prog. Opt. 55, 85 (2010).
[36] K. P. Heeg et al., Vacuum-Assisted Generation and Control of Atomic Coherences at X-Ray Energies, Phys. Rev. Lett. 111, 073601 (2013).

[37] H. Freedhoff and T. Quang, Ultrasharp Lines in the Absorption and Fluorescence Spectra of an Atom in a Cavity, Phys. Rev. Lett. 72, 474 (1994).

[38] M. B. Plenio and P. L. Knight, The quantum-jump approach to dissipative dynamics in quantum optics, Rev. Mod. Phys. 70, 101 (1998).

[39] F. Verstraete, M. M. Wolf, and J. Ignacio Cirac, Quantum computation and quantum-state engineering driven by dissipation, Nat. Phys. 5, 633 (2009). 